\title{
Interaction between rs10830963 polymorphism in MTNR1B and lifestyle intervention on occurrence of gestational diabetes
}

\author{
Nora E. Grotenfelt ${ }^{1}$ • Niko S. Wasenius ${ }^{1,2} \cdot$ Kristiina Rönö $^{3} \cdot$ Hannele Laivuori $^{3,4,5}$ • \\ Beata Stach-Lempinen ${ }^{6}$ • Marju Orho-Melander ${ }^{7}$. Christina-Alexandra Schulz ${ }^{7}$. \\ Hannu Kautiainen $^{2,8}$ • Saila B. Koivusalo ${ }^{3}$. Johan G. Eriksson ${ }^{1,2,9}$
}

Received: 2 February 2016 /Accepted: 21 April 2016/Published online: 21 May 2016

(C) Springer-Verlag Berlin Heidelberg 2016

\begin{abstract}
Aims/hypothesis The aim of this study was to assess the interaction between melatonin receptor 1B gene (MTNR1B) rs10830963 polymorphism and lifestyle intervention during pregnancy on occurrence of gestational diabetes mellitus (GDM) in high-risk women.

Methods This is a secondary analysis of the randomised controlled gestational diabetes prevention trial 'RADIEL', conducted between 2008 and 2014 in four maternity hospitals in southern Finland. A total of 226 women with a history of GDM and/or a pre-pregnancy $\mathrm{BMI} \geq 30 \mathrm{~kg} / \mathrm{m}^{2}$ were enrolled
\end{abstract}

Nora E. Grotenfelt

nora.grotenfelt@helsinki.fi

1 Folkhälsan Research Centre, Biomedicum Helsinki, University of Helsinki, Haartmaninkatu 8, P.O. Box 63, 00014 Helsinki, Finland

2 Department of General Practice and Primary Health Care, University of Helsinki and Helsinki University Hospital, Helsinki, Finland

3 Department of Obstetrics and Gynecology, University of Helsinki and Helsinki University Hospital, Helsinki, Finland

4 Institute for Molecular Medicine Finland, University of Helsinki, Helsinki, Finland

5 Department of Medical and Clinical Genetics, University of Helsinki and Helsinki University Hospital, Helsinki, Finland

6 Department of Obstetrics and Gynecology, South-Karelia Central Hospital, Lappeenranta, Finland

7 Department of Clinical Sciences in Malmö, Lund University Diabetes Center, Lund University, Lund, Sweden

8 Department of General Practice and Primary Health Care, University of Eastern Finland, Kuopio, Finland

9 Department of Chronic Disease Prevention, National Institute for Health and Welfare, Helsinki, Finland at $<20$ weeks of gestation (mean 13 weeks) and randomised into an intervention group receiving counselling on diet, physical activity and weight control and a control group receiving standard antenatal care. The main outcome was incidence of GDM, defined as one or more pathological glucose values in a standard $75 \mathrm{~g}$ 2-h OGTT. The MTNR1B rs10830963 was genotyped for further analyses.

Results No significant differences were found in the genotype distribution between the intervention and the control group. A significant interaction was observed between the rs 10830963 genotypes and the lifestyle intervention on age-adjusted occurrence of gestational diabetes $(p=0.038)$. Among women homozygous for the $\mathrm{C}$ allele of rs 10830963 , the OR for GDM was significantly lower in the intervention group than in the control group (OR 0.16 [95\% CI 0.03, 0.85], $p=0.014$ ). This difference was not seen in women heterozygous (OR 0.88 [95\% CI 0.32, 2.41], $p=0.798$ ) or homozygous (OR 2.25 [95\% CI 0.34, 14.69], $p=0.384$ ) for the risk allele $\mathrm{G}$.

Conclusions/interpretation In women at high risk of GDM, only those not carrying the risk allele $G$ benefited from the lifestyle intervention. Our results indicate that certain genetic risk variants may modify the effectiveness of lifestyle interventions. This may provide important information when planning GDM prevention studies in the future.

Keywords Clinical trial · Diabetes · Gestational · Melatonin · MT2 · Polymorphism · Pregnancy · Prevalence · Public health $\cdot$ Receptor $\cdot$ Single nucleotide
Abbreviations
GDM Gestational diabetes mellitus
MTNR1B Melatonin receptor 1B 


\section{Introduction}

Worldwide, the incidence of gestational diabetes (GDM) is increasing [1], as is evidence regarding the adverse effects of GDM on the short- and long-term health outcomes of both the mother and the offspring [2]. Evidence is accumulating regarding the role of genetic factors in the aetiology of GDM [3] together with the well-known clinical risk factors overweight and obesity.

Studies assessing the common etiological factors of type 2 diabetes and GDM have revealed associations between GDM and genetic susceptibility loci for type 2 diabetes [3]. Among these, the risk allele $(\mathrm{G})$ of rs10830963 in the melatonin receptor 1B (MTNR1B) gene (MTNR1B) shows the highest OR for GDM in a Finnish study [4].

MTNR1B is one of the seven transmembrane G-proteincoupled melatonin receptors expressed in the central nervous system and in the peripheral tissues including the pancreatic beta cells [5].

The hormone melatonin is mainly secreted from the pineal gland in response to darkness in a diurnal fashion [6]. It mediates the involvement of circadian rhythms in physiological functions mostly by binding to the MTNR1A or MTNR1B isoforms. Evidence links melatonin with decreased insulin secretion [6].

The results of several intervention trials aimed at reducing GDM have been inconsistent and discouraging [7]. We hypothesised that these inconsistent results may in part be due to genetic factors, such as the rs 10830963 polymorphism in MTNR1B.

To investigate whether genetic variation in MTNRIB may modify the GDM outcome of a lifestyle intervention, we genotyped the rs10830963 in the GDM prevention trial (RADIEL), targeting women at high risk for GDM [8]. To our knowledge, no studies have assessed the interaction between genetic variants and the outcome of GDM prevention.

\section{Methods}

This is a secondary analysis of the randomised controlled GDM prevention trial 'RADIEL' (ClinicalTrial.gov registration no. NCT01698385), conducted between February 2008 and January 2014 in four maternity hospitals in southern Finland and approved by the Ethics Committee of Helsinki University Central Hospital. A total of 269 women over 18 years of age with a history of GDM and/or a prepregnancy $\mathrm{BMI} \geq 30 \mathrm{~kg} / \mathrm{m}^{2}$ and no overt diabetes at study inclusion were enrolled at $\leq 20$ weeks of gestation (on average 13 weeks) and randomised into an intervention group $(n=144)$ receiving counselling on diet and physical activity and a control group $(n=125)$ receiving standard antenatal care. All study participants gave informed consent. The main outcome was incidence of GDM, defined according to ADA 2008 criteria as one or more pathological glucose values in a $75 \mathrm{~g}$ 2-h OGTT at 24-28 weeks of gestation.

The design, the power calculation and the main results of the RADIEL intervention trial have been published previously [8].

DNA was extracted from whole blood samples using Qiagen Maxipreps (Qiagen, Valencia, CA, USA). Rs10830963 was genotyped using a Sequenom iPLEX platform (Sequenom, San Diego, CA, USA). Rs10830963 was successfully genotyped in 226 participants. The genotypes were in HWE ( $p=0.30$ ), genotyping success rate was $84 \%$, and repeated genotyping was performed in a random sample of $5 \%$ of the participants with a concordance rate of $100 \%$.

HOMA-IR was calculated using computer-generated HOMA2 index (www.dtu.ox.ac.uk, assessed 5 November 2014).

Data are reported as means (SD) or counts (percentages). The comparison of the baseline characteristics between the different rs 10830963 genotype carriers was made using $\chi^{2}$ test or regression analysis with pre-defined analysis of variance type contrasts and Bonferroni corrected post hoc tests. Logistic regression analysis was applied to assess unadjusted and age-adjusted association between rs 10830963 and GDM. The age-adjusted interaction between rs10830963 and lifestyle intervention on GDM was assessed using logistic regression models including the main effects of genotype and intervention group, and their multiplicative term. Bootstrap type analysis with 10,000 replications was applied in the case of violation of assumptions. Significance: $p$ values $<0.05$ were considered to be statistically significant. Analyses were performed with Stata/SE 13.1 (StataCorp, TX, USA). Simulations were used to calculate post hoc power for the interaction term by using the actual data from this study.

\section{Results}

The minor allele frequency was 0.34 and no significant differences in genotype frequencies existed between the intervention groups. At baseline, the $\mathrm{G}$ allele was associated with a lower pre-pregnancy BMI $(p=0.03)$, insulin $(p=0.04)$, HOMA-IR $(p<0.005)$, total cholesterol $(p=0.02)$, LDLcholesterol $(p<0.01)$ and with higher HDL-cholesterol $(p<0.005)$ (Table 1).

In total, GDM occurred in $17 \%$ of all 226 participants, and rs10830963 was associated with overall occurrence of GDM (unadjusted OR 1.82 [95\% CI 1.10, 3.02], $p=0.019$ ). This association remained significant after adjustment for age (OR 1.77, 95\% CI 1.07, 2.92, $p=0.026$ ).

An interaction ( $p=0.038$, post hoc power $64 \%$ ) appeared between rs10830963 and the lifestyle intervention on ageadjusted occurrence of GDM. The rs10830963 genotype 
Table 1 Genotype frequencies of MTNR1B polymorphism rs10830963. Clinical characteristics at baseline according to genotype in 226 participants from the RADIEL trial

\begin{tabular}{|c|c|c|c|c|c|c|c|c|}
\hline & Total, $n=226$ & $\mathrm{CC} n=103$ & $\mathrm{CG} n=94$ & GG $n=29$ & $p$ value $^{\mathrm{a}}$ & $\begin{array}{l}p \text { value for } \\
\mathrm{CC} / \mathrm{CG}\end{array}$ & $\begin{array}{l}p \text { value for } \\
\mathrm{CC} / \mathrm{GG}\end{array}$ & $\begin{array}{l}p \text { value for } \\
\mathrm{CG} / \mathrm{GG}\end{array}$ \\
\hline $\begin{array}{l}\text { Genotype frequency } \\
(\%)\end{array}$ & & 46 & 42 & 13 & & & & \\
\hline $\begin{array}{l}\text { Intervention group, } \mathrm{I} / \mathrm{C}^{\mathrm{b}} \\
n,(\%)\end{array}$ & & $55(46) / 48(45)$ & 47 (39) / 47 (44) & $18(15) / 11(10)$ & 0.52 & & & \\
\hline Age (years) & $32.5(4.6)$ & $32.1(4.5)$ & $32.4(4.7)$ & $34.0(4.7)$ & 0.15 & 1.00 & 0.16 & 0.35 \\
\hline Gestational weeks & $23.0(1.4)$ & $23.1(1.3)$ & $22.9(1.5)$ & $22.8(1.3)$ & 0.48 & 1.0 & 0.81 & 1.00 \\
\hline Pre-pregnancy BMI (kg/m²) & $31.7(5.8)$ & $32.8(5.3)$ & $30.9(6.0)$ & $30.4(6.0)$ & 0.03 & 0.06 & 0.16 & 1.00 \\
\hline Weight $(\mathrm{kg})$ & $89.4(17.0)$ & $91.5(15.0)$ & $87.5(18.5)$ & $87.7(18.4)$ & 0.22 & 0.31 & 0.92 & 1.00 \\
\hline Systolic BP (mmHg) & $121.5(13.3)$ & $122.8(13.3)$ & $120.5(12.1)$ & $120.2(16.4)$ & 0.44 & 0.68 & 1.00 & 1.00 \\
\hline Diastolic BP (mmHg) & $77.4(8.9)$ & $77.0(8.7)$ & $77.6(8.5)$ & $78.3(10.5)$ & 0.75 & 1.00 & 1.00 & 1.00 \\
\hline $\begin{array}{l}\text { Fasting plasma } \\
\text { glucose }(\mathrm{mmol} / \mathrm{l})\end{array}$ & $4.9(0.2)$ & $4.9(0.2)$ & $4.9(0.2)$ & $4.9(0.3)$ & 0.85 & 1.00 & 1.00 & 1.00 \\
\hline $\mathrm{HbA}_{1 \mathrm{c}}(\%)$ & $5.2(0.27)$ & $5.2(0.26)$ & $5.2(0.31)$ & $5.2(0.22)$ & & & & \\
\hline $\mathrm{HbA}_{1 \mathrm{c}}(\mathrm{mmol} / \mathrm{mol})$ & $33.5(3.0)$ & $33.5(2.8)$ & $33.7(3.4)$ & $32.8(2.4)$ & 0.33 & 1.00 & 0.56 & 0.47 \\
\hline Insulin (pmol/1) & $51.5(39.8)$ & $55.9(25.9)$ & $46.0(26.8)$ & $54.3(89.0)$ & 0.04 & 0.04 & 1.00 & 1.00 \\
\hline HOMA-IR & $1.1(0.5)$ & $1.2(0.5)$ & $1.0(0.6)$ & $0.9(0.4)$ & $<0.005$ & 0.13 & $<0.005$ & 0.24 \\
\hline Total cholesterol (mmol/l) & $4.9(0.9)$ & $5.0(1.0)$ & $4.7(0.8)$ & $5.0(0.7)$ & 0.02 & 0.02 & 1.00 & 0.21 \\
\hline HDL-cholesterol (mmol/l) & $1.7(0.3)$ & $1.7(0.3)$ & $1.8(0.4)$ & $1.8(0.2)$ & $<0.005$ & 0.10 & $<0.005$ & 0.61 \\
\hline LDL-cholesterol (mmol/l) & $2.8(0.7)$ & $3.0(0.8)$ & $2.6(0.6)$ & $2.7(0.6)$ & $<0.01$ & $<0.005$ & 0.29 & 1.00 \\
\hline Total triacyglycerol (mmol/l) & $1.3(0.7)$ & $1.4(0.6)$ & $1.3(0.7)$ & $1.3(0.6)$ & 0.45 & 0.63 & 1.00 & 1.00 \\
\hline
\end{tabular}

Data are mean (SD) unless otherwise stated

${ }^{a}$ Associations between the clinical characteristics at baseline and MTNR1B rs 10830963 genotypes were tested using ANOVA (continuous variable) and $X^{2}$ test (categorical variables)

${ }^{\mathrm{b}} \mathrm{I}$, intervention group; $\mathrm{C}$, control group

was positively associated with occurrence of GDM in the intervention group ( $p$ for linearity $=0.003$ ) but not in the control group ( $p$ for linearity $=0.991)$. Among women homozygous for the C allele of rs10830963, risk for GDM was significantly lower in the intervention group than in the control group (OR 0.16 [95\% CI 0.03, 0.85], $p=0.014$ ). This difference was not seen in women heterozygous (OR 0.88 [95\% CI $0.32,2.41], p=0.798$ ) or homozygous (OR 2.25 [95\% CI $0.34,14.69], p=0.384$ ) for the risk allele $\mathrm{G}$ (Fig. 1).

\section{Discussion}

Among women at high risk for GDM, only those not carrying the rs 10830963 risk allele $G$ seem to benefit from the RADIEL lifestyle intervention. Our results suggest that the risk for GDM associated with one or two copies of the rs 10830963 risk allele $\mathrm{G}$ is not modifiable by the otherwise successful lifestyle intervention in the RADIEL study.

Melatonin and its receptor MTNR1B together play an important role in glucose homeostasis. The risk G allele of rs10830963 in MTNR1B has been associated with elevated fasting glucose levels and with decreased early insulin secretion in response to glucose [5], as well as with decreased insulin sensitivity [9]. In addition, the risk allele has also been associated with increased expression of MTNRIB in pancreatic beta cells, thereby possibly enhancing the melatonin effect of impaired early insulin secretion [5].

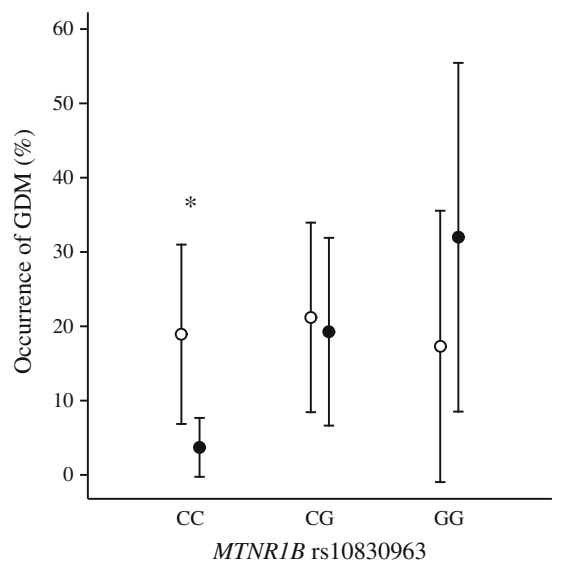

Fig. 1 Association between MTNR1B rs10890363 polymorphism and age-adjusted occurrence of GDM in the control group (white circles, $p$ for linearity $=0.991$ ) and in the lifestyle intervention group (black circles, $p$ for linearity $=0.003)(p$ for interaction $=0.038)$ in 226 women participating in the RADIEL trial. Error bars indicate $95 \%$ CIs 
The $\mathrm{G}$ allele carriers were less insulin resistant at baseline and at higher risk of GDM during follow-up. The mechanism behind this may be an effect of the risk allele on the insulin production documented in several previous studies. The success of a lifestyle intervention aiming at preventing GDM is probably mediated mainly through the diminishing of increased insulin resistance. As the main reason for increased GDM risk among the $\mathrm{G}$ allele carriers seems to be impaired insulin production, we hypothesise that they may not benefit as much as the non-risk allele carriers from a reduced increase in insulin resistance.

The presence of risk alleles in different susceptibility loci might at least partly explain the inconsistent results of previous GDM prevention trials. Interestingly, the rs10830963 associates with the outcome of an intervention that targets weight loss [10]. The mechanism linking risk allele presence with intervention failure has yet to be determined. Further studies should investigate whether other susceptibility loci affect the outcome of lifestyle interventions. This knowledge may improve the targeting and cost-effectiveness of future GDM prevention strategies.

The strength of this study lies in its unusual design, adding to the knowledge of factors that may affect the result of GDM prevention interventions. It would be highly interesting to apply new diagnostic criteria to this study. However, we consider re-analysing the data according to the IADPSG 2010 definition of GDM inappropriate since the design of this study is based on the 2008 ADA definition. Applying the IADPSG 2010 definition of GDM in retrospect is not possible since it would affect the inclusion criteria. Weaknesses of the study include generalisability of the findings; in populations with different ethnic backgrounds the role of MTNR $1 B$ rs10830963 may be of lesser importance than in this genetically relatively homogenous Finnish population. In addition, the small sample size, the lack of power and the lack of replication data suggest that the findings should be interpreted with caution.

Our findings imply that genetic risk factors need further evaluation, and that they should be taken into account to optimise the targeting of GDM prevention strategies.

Funding The study is funded by Ahokas Foundation, Finnish Foundation for Cardiovascular Disease, Special state subsidy for health science research of Helsinki University Hospital, Samfundet Folkhälsan, Finska Läkaresällskapet, Liv och Hälsa, The Finnish Diabetes Research Foundation, State Provincial Office of Southern Finland, The Social Insurance Institution of Finland, The Swedish Research Council, The Swedish Heart and Lung Foundation and The Novo Nordisk Foundation.

The funders have not had any role in designing or conducting the study; nor in collection, management, analysis, or interpretation of the data; nor in preparation, review, or approval of the manuscript; and decision to submit the manuscript for publication.
Duality of interest The authors declare that there is no duality of interest associated with this manuscript.

Contribution statement NEG participated in the implementation of the study, literature search, data interpretation and the drafting and editing of the article. NSW performed the statistical analyses, participated in the data interpretation and the drafting and editing of the article. KR participated in the implementation of the study, prepared the database and participated in the drafting of the article. HL participated in the design of the study and the drafting of the article. BS-L participated in the design of the study, coordinated the study in Lappeenranta and participated in the drafting of the article. MO-M and C-AS performed the genotyping, helped with data interpretation and revised the manuscript critically for important intellectual content. HK helped with the statistical analyses and the drafting and editing of the article. SBK initiated, participated in the design of, and coordinated the study; and helped in the drafting and editing of the article. JGE is the principal investigator of the study, and participated in the implementation of the study and advised on editing the article. All authors have read and approved the final version of the manuscript. NEG is the guarantor of this work and, as such, had full access to all the data in the study and takes responsibility for the integrity of the data and the accuracy of the data analysis.

\section{References}

1. Ferrara A (2007) Increasing prevalence of gestational diabetes mellitus: a public health perspective. Diabetes Care 30(Suppl 2): S141-S146

2. Catalano PM, McIntyre HD, Cruickshank JK et al (2012) The Hyperglycemia and Adverse Pregnancy Outcome Study: associations of GDM and obesity with pregnancy outcomes. Diabetes Care 35:780-786

3. Lowe WL Jr, Karban J (2014) Genetics, genomics and metabolomics: new insights into maternal metabolism during pregnancy. Diabet Med 31:254-262

4. Huopio H, Cederberg H, Vangipurapu J et al (2013) Association of risk variants for type 2 diabetes and hyperglycemia with gestational diabetes. Eur J Endocrinol 169:291-297

5. Lyssenko V, Nagorny CL, Erdos MR et al (2009) Common variant in MTNR1B associated with increased risk of type 2 diabetes and impaired early insulin secretion. Nat Genet 41:82-88

6. Peschke E, Bahr I, Muhlbauer E (2013) Melatonin and pancreatic islets: interrelationships between melatonin, insulin and glucagon. Int J Mol Sci 14:6981-7015

7. Oteng-Ntim E, Varma R, Croker H, Poston L, Doyle P (2012) Lifestyle interventions for overweight and obese pregnant women to improve pregnancy outcome: systematic review and metaanalysis. BMC Med 10:47. doi:10.1186/1741-7015-10-47

8. Koivusalo SB, Rono K, Klemetti MM et al (2016) Gestational diabetes mellitus can be prevented by lifestyle intervention: The Finnish Gestational Diabetes Prevention Study (RADIEL): a randomized controlled trial. Diabetes Care 39:24-30

9. Jonsson A, Ladenvall C, Ahluwalia TS et al (2013) Effects of common genetic variants associated with type 2 diabetes and glycemic traits on alpha- and beta-cell function and insulin action in humans. Diabetes 62:2978-2983

10. Goni L, Cuervo M, Milagro FI, Martinez JA (2014) Gene-gene interplay and gene-diet interactions involving the MTNR1B rs10830963 variant with body weight loss. J Nutrigenet Nutrigenomics 7:232-242 